\title{
Simplifying topological entanglements by entropic competition of slip-links
}

\author{
Andrea Bonato $\odot,{ }^{1}$ Davide Marenduzzo, ${ }^{1}$ and Davide Michieletto ${ }^{1,2}$ \\ ${ }^{1}$ University of Edinburgh, SUPA, School of Physics and Astronomy, Peter Guthrie Road, Edinburgh EH9 3FD, United Kingdom \\ ${ }^{2}$ MRC Human Genetics Unit, Institute of Genetics and Molecular Medicine, University of Edinburgh, Edinburgh EH4 2XU, United Kingdom
}

(Received 4 October 2020; accepted 24 August 2021; published 25 October 2021)

\begin{abstract}
Topological entanglements are abundant, and often detrimental, in polymeric systems in biology and materials science. Here we theoretically investigate the topological simplification of knots by diffusing slip-links (SLs), which may represent biological or synthetic molecules, such as structural maintenance of chromosome proteins or cyclodextrins in slide-ring gels. We find that SLs entropically compete with knots and can localize them, greatly facilitating their downstream simplification by transient strand-crossing. We further show that the efficiency of knot localization depends strongly on the topology of the SL network, and, informed by our findings, we discuss potential strategies to control the topology of biological and synthetic materials.
\end{abstract}

DOI: 10.1103/PhysRevResearch.3.043070

\section{INTRODUCTION}

Knots and topological entanglements are often found in physical and biological systems [1-4]. Their uncontrolled formation and proliferation reduces the space of accessible configurations of generic polymer chains, in turn potentially affecting their mechanical [5] or biological [6-8] functions. Entanglements are so inevitable and detrimental in the genome of living organisms that a specific class of highly conserved proteins - known as topoisomerases-has evolved to resolve them [9].

While it is typical to treat abundant topological constraints at the mean-field level-for instance in the tube model of polymer melts [10] - exact and scaling results can be obtained via theories that replace entanglements with slip-links (SLs), which enforce contacts between polymer segments while allowing them to slide past each other [11-15]. Because of this physically appealing analogy, systems of polymers with slip-links have been theoretically and numerically explored in the field of statistical and polymer physics, for instance to estimate the size of knots $[16,17]$ and the effective tube size in polymer melts [18].

Beyond the theoretical appeal of these systems, actual examples in which SLs affect the conformation of polymers can be found in nature and materials science. For instance, the family of structural maintenance of chromosome (SMC) proteins act as SLs on the genome, and, depending on the condition and organism, they can either actively move [19-22] or passively diffuse [23-26], in turn extruding loops. These proteins are key for the organization of the genome in vivo

Published by the American Physical Society under the terms of the Creative Commons Attribution 4.0 International license. Further distribution of this work must maintain attribution to the author(s) and the published article's title, journal citation, and DOI.
[27-29], for the structure of sister chromatids [29,30], and to regulate chromatid decatenation and cell division [31]. Additionally, it was recently suggested that they cooperate with topoisomerases to keep the genome topologically simple and entanglement-free [32]. While the structural role of SMC proteins is now widely explored, the mechanisms through which they regulate genome topology are far less understood or investigated. At the same time, SLs can be realized in synthetic and supramolecular chemistry using cyclodextrins [33] and are employed for instance to make molecular machines [34] and slide-ring gels [35]. In these cases, the benefit of using SLs is that they effectively act as mobile cross-links, thus imposing strong, yet plastic, topological constraints on the polymeric substrates. Thanks to this peculiar feature, gels of polyrotaxanes display toughness and self-healing abilities far superior to those of traditionally cross-linked materials [36]. As in biological systems, also the SL-mediated topological regulation of synthetic slide-ring gels is only starting to be investigated [37].

In this work, we study the interplay between SLs and topological entanglements formed by knots tied on a ring polymer (see Fig. 1). While our setup is inspired by the existing framework of entropic competition [16,17,38-41], here we consider SLs as real components of the system, modeling the presence of (diffusing) SMC proteins or cyclodextrins. In particular, we compute the loop size distribution and length of knotted segments for a variety of SL network topologies, and we show that diffusing SLs are able to localize knotted segments purely by entropy, without any external energy input. Importantly, and in marked contrast with previous works on entropic competition, we show that the efficiency of entanglement localization depends on the particular topology of the SL network, and that including the action of topoisomerases (modeled as transient strand-crossings) leads to extremely fast and efficient simplification of complex knots. Our results suggest an entropy-driven mechanism through which generic SL-like molecules can regulate the topology of DNA or synthetic polymers. 


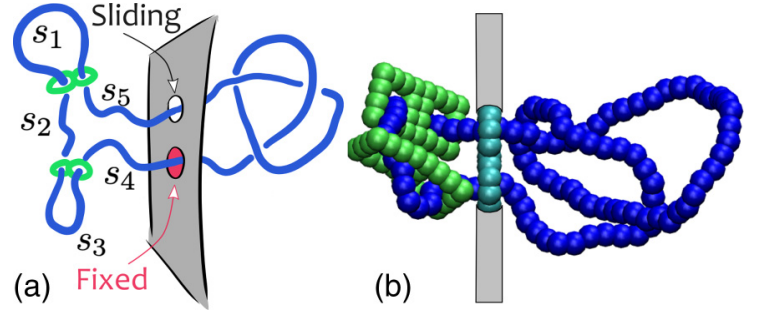

FIG. 1. Entropic competition of SL and entanglements. (a) Our setup is inspired by that of entropic competition [16]: a ring polymer with total length $L$ is tied in a knot and contains diffusing SLs. The ring is forced to pass through two holes on a wall that separates the two sides. Only the contour passing through the top hole is allowed to slide, while the other is fixed. In the figure, the left-handed loop has $N_{\mathrm{SL}}=2 \mathrm{SLs}$ and is partitioned into $2 N_{\mathrm{SL}}+1=5$ segments, of which $s_{1}$ and $s_{3}$ are called peripheral loops; $s_{2}, s_{4}$, and $s_{5}$ are called internal loops; and $s_{2}+s_{4}+s_{5}$ makes up the inner loop. The righthand side contains a $3{ }_{1}$ knot. (b) Snapshot from molecular dynamics simulations of the system in (a).

\section{MODEL AND METHODS}

We prepare the initial configuration of our system by joining two bead-spring polymer segments on either side of a wall; typically, we consider configurations in which the two polymer segments are either knotted or contain SLs. Since in our model the polymer cannot cross the wall, itself, or the SLs, the topology on each side is preserved throughout the simulations (see below).

More specifically, we perform molecular dynamics simulations of bead-and-spring polymers made up of beads of size $\sigma$. The simulation box is separated into two equally large portions by an impenetrable wall, and each bead interacting with the wall is subject to a repulsive force perpendicular to the wall described by the potential:

$$
U_{\text {wall }}(r)= \begin{cases}\epsilon\left[\frac{2}{15}\left(\frac{\sigma}{r}\right)^{9}-\left(\frac{\sigma}{r}\right)^{3}+\frac{2}{3} \sqrt{\frac{5}{2}}\right], & r \leqslant r_{c}, \\ 0, & r>r_{c},\end{cases}
$$

where $r$ is the separation between the bead and the wall, and $r_{c}=\left(\frac{2}{5}\right)^{\frac{1}{6}} \sigma$. Beads of the polymer are strung together by finite-extension-nonlinear-elastic (FENE) bonds:

$$
U_{\mathrm{FENE}}(r)= \begin{cases}-0.5 k R_{0}^{2} \ln \left[1-\left(r / R_{0}\right)^{2}\right], & r \leqslant R_{0}, \\ \infty, & r>R_{0},\end{cases}
$$

where $R_{0}=1.5 \sigma$ is the maximum extension of the bond; interactions between beads are governed by the WeeksChandler-Andersen (WCA) potential:

$$
U_{\mathrm{WCA}}(r)= \begin{cases}4 \epsilon\left[\left(\frac{\sigma}{r}\right)^{12}-\left(\frac{\sigma}{r}\right)^{6}+\frac{1}{4}\right], & r \leqslant r_{c}, \\ 0, & r>r_{c},\end{cases}
$$

where $r$ is the distance between the bead centers and $r_{c}=$ $2^{1 / 6} \sigma$. To account for the effect of stiffness (arising, for instance, if the polymers represent chromatin fibres), we introduced a Kratky-Porod potential acting on triplets of neighboring beads:

$$
U_{\text {bend }}=\frac{k_{B} T l_{p}}{\sigma}(1+\cos \theta),
$$

where $\theta$ is the angle between consecutive bonds. The initial configurations are designed such that a polymer passes through two holes in a wall, the size of which is small enough to let just one monomer through at a time. In this way, the polymer is divided into two segments, physically separated by the wall. Additionally, we fix the position of one of the two beads closest to the wall so that the segments can exchange monomers only through the other hole. This setup is reminiscent of the one used to study entropic competition in Ref. [41].

Slip-links (SLs) are modeled as physical square handcuffs and are allowed to slide diffusively along the polymer. The setup is such that the polymer passes through two holes on the wall, the size of which is small enough to let just one monomer through each of them at any time (see Fig. 1). Specifically, we consider SLs with side $4 \sigma$, held together by four FENE bounds and opened in a "planar" arrangement [this is achieved with the potential described by Eq. (4) and large enough $l_{p}$ ].

To study the kinetics of topological simplification later in this work, we allow a short (10 beads) segment to undergo strand-crossings. This is achieved by setting to 0 the amplitude of the (WCA) potential regulating the interaction between the beads of this segment and each bead composing the rest of the polymer, excluding the two immediately next to the segment boundaries. In our slip-link versus knot simulations, this segment is kept on the knot side by placing it next to the fixed bead (wall).

\section{Sampling of the system}

To make sure that we are sampling our system ergodically and that we are avoiding kinetic traps that may exist in MD simulations, we take snapshots of our system every $10^{6}$ LAMMPS time steps $\left(10^{4} \tau_{B}\right)$, comparable to the decorrelation time of a polymer of length up to at least 200 monomers. Within this time also the SLs loaded onto the substrate have traveled a significant amount of contour length [42], and so we assume that each sampled configuration is independent of others. Some of our results obtained with polymers of length 500 may thus display longer decorrelation times. In Fig. 2 we plot the radius of gyration $R_{g}(t)$ of a polymer and the autocorrelation function $G_{O}(t)$ of a scalar observable $O$ of the polymer as a function of time. These are estimated as

$$
R_{g}^{2}(t)=\frac{1}{L} \sum_{i=1}^{L}\left[\mathbf{r}_{i}(t)-\langle\mathbf{r}(t)\rangle\right]^{2},
$$

where $\mathbf{r}_{\mathbf{i}}$ is the position of the $i$ th bead, $L$ is the length of the polymer, $\langle\mathbf{r}(t)\rangle=\frac{1}{L} \sum_{i=1}^{L} \mathbf{r}_{\mathbf{i}}(t)$, and

$$
G_{O}(t)=\frac{1}{(T-t) \sigma_{O}^{2}} \sum_{i=1}^{T-t}[O(i)-\langle O\rangle][O(i+t)-\langle O\rangle],
$$

where $T$ is the number of sampled configurations, and $O(i)$ is the value of $O$ for the $i$ th configuration, $\langle O\rangle=\frac{1}{T} \sum_{i=1}^{T} O(i)$ and $\sigma_{O}^{2}=\frac{1}{T} \sum_{i=1}^{T}(O(i)-\langle O\rangle)^{2}$. By comparing Figs. 2(b) and 2(f), one can appreciate that the 500-bead-long chain displays a slower decay of the autocorrelation of the length of side 2 $\left[G_{l_{2}}(t)\right]$. This autocorrelation appears to decay around $10^{5} \tau_{B}$ while we sample the system every $10^{4} \tau_{B}$ time steps. 


\section{$\mathrm{L}=100 \quad \mathrm{~L}=500$}
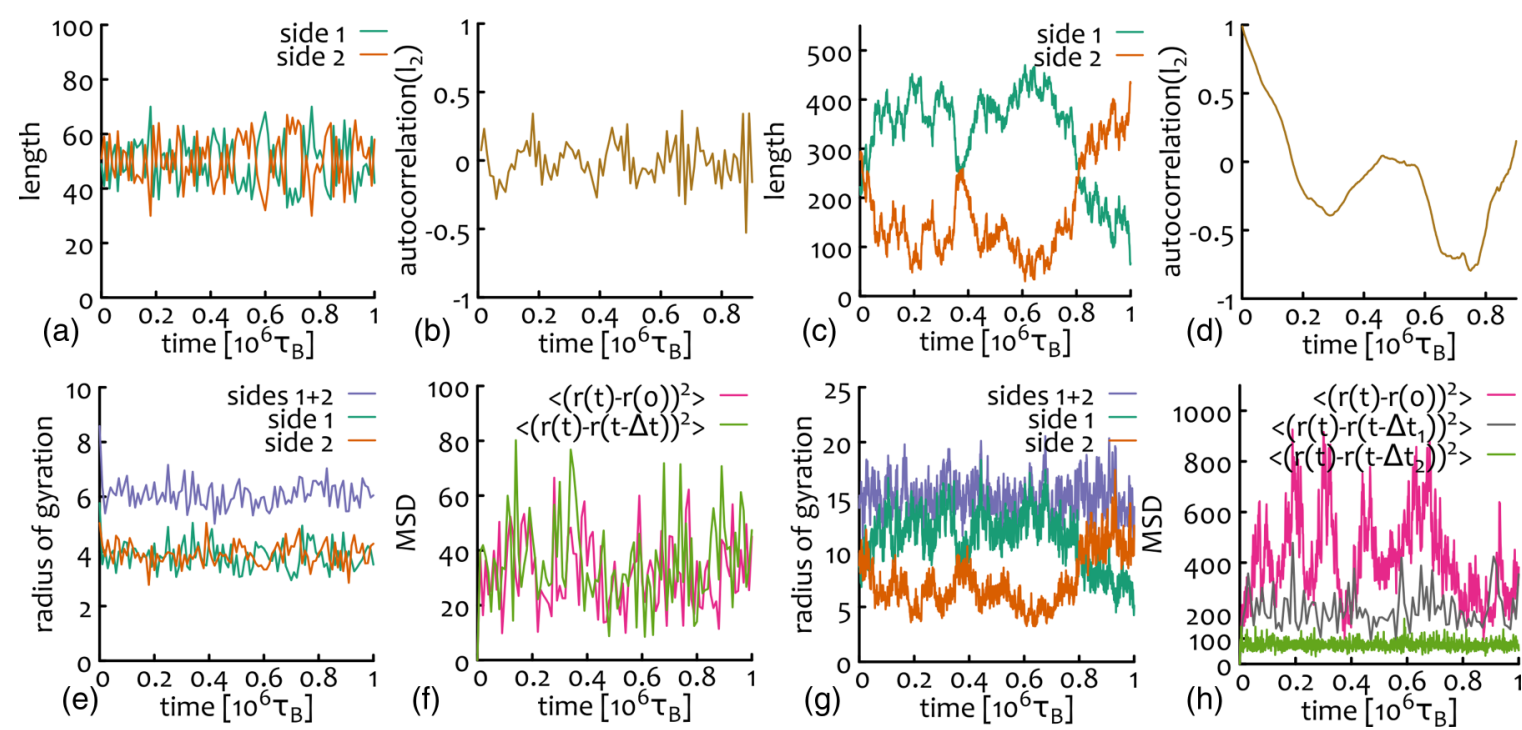

FIG. 2. Conformational decorrelation time of a 100-bead long (left) and 500-bead long (right) polymer in the 2SLs vs 2SLs configuration. (a),(c) Length of the two competing sides of the polymer as a function of time. (b),(d) Normalized autocorrelation function of the length of one of the two competing sides (labeled as side 2). (e),(g) Radius of gyration in units of $\sigma$ as a function of time. (f),(h) Mean-squared displacement in units of $\sigma^{2}$ of a bead of the polymer as a function of time. $\Delta t=\Delta t_{1}=10^{4} \tau_{B} ; \Delta t_{2}=10^{3} \tau_{B}$.

\section{COMPETITION OF SLS}

We first study the competition between symmetric SL networks: we load up to three SLs on each side of the wall in either a "round-table" (RT) configuration, realized by loading the slip-links in series [16] [Figs. 3(a), 3(c) and 3(d)], or a "necklace" (NC) configuration, characterized by nested loops [16] [Fig. 3(b)]. The number of configurations of a ring of length $L$ containing $N_{\mathrm{SL}}$ SLs in the RT configuration or two SLs in the NC configuration, and grafted to an impenetrable surface as in Fig. 3, scales as (see Appendix A)

$$
\begin{aligned}
\mathcal{Z}_{\mathrm{RT}, N_{\mathrm{SL}}}(L) \sim & \int d s_{1} \int d s_{2} \cdots \int d s_{2 N_{\mathrm{SL}}+1} \\
& \times\left[\mu^{L}\left(L-\sum_{i=1}^{N_{\mathrm{SL}}} s_{i}\right)^{-3 v-1} \prod_{i=1}^{N_{\mathrm{SL}}} s_{i}{ }^{\alpha}\right]_{\sum_{i=1}^{2 N_{\mathrm{SL}}+1} s_{i}=L},
\end{aligned}
$$

where $s_{i}, i=1, \ldots, N_{\mathrm{SL}}$ are the lengths of the $N_{\mathrm{SL}}$ peripheral loops, $s_{i}, i=N_{\mathrm{SL}}+1, \ldots, 2 N_{\mathrm{SL}}+1$ are the lengths of the other $N_{\mathrm{SL}}+1$ segments (see Fig. 1; note that the wall is not considered as a SL), $v$ is the universal metric exponent relating the size of a polymer to its length, and $\mu$ is the nonuniversal connectivity constant. By using known results for the statistical physics of polymer networks [16,43], $\alpha$ can be computed as

$$
\alpha=-3 v+\sigma_{4},
$$

with $\sigma_{4}=-0.46$ the scaling exponent associated with fourlegged nodes [43]. The integration extends over all lengths compatible with the given topology, and accounts for the sliding entropy of the SLs. Since the two competing slip-link networks in Fig. 3 are not interacting, the total number of configurations can be factorized as the product of the number of configurations on each side.
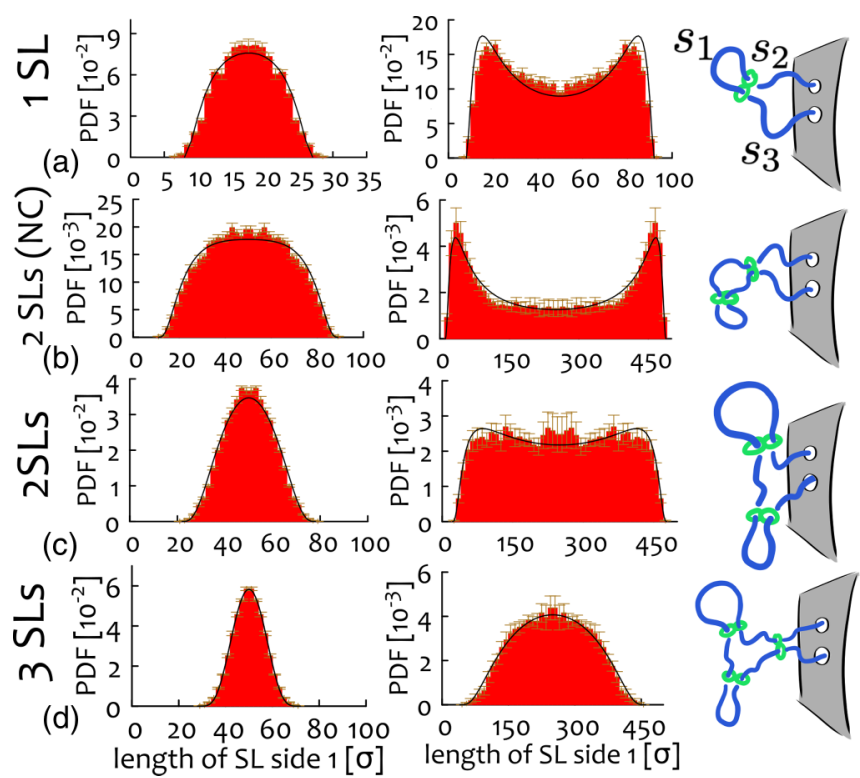

FIG. 3. Entropic competition between symmetric SL networks. From top to bottom: (a) a single SL; (b) two SLs in a necklace configuration; (c) two SLs in a round-table configuration; (d) three SLs in a round-table configuration. Note that we simulate two SL networks with identical topology, one on each side of the wall. Each histogram collects information from $10^{4}$ configurations sampled every $10^{6}$ LAMMPS steps from 100 independent replicas. The black curves are obtained solving Eq. (7) for the relevant SL network topology. From left to right: increasing total length of the ring polymer [35, 100 beads in (a) and 100, 500 beads in (b)-(d)]. 


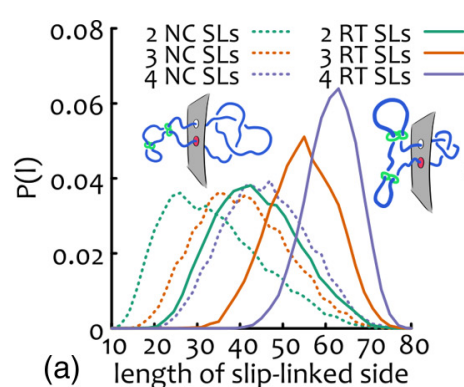

(a) length of slip-linked side

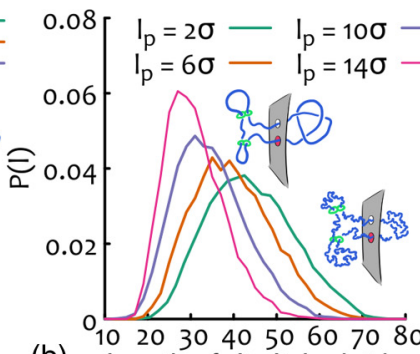

(b) length of slip-linked side

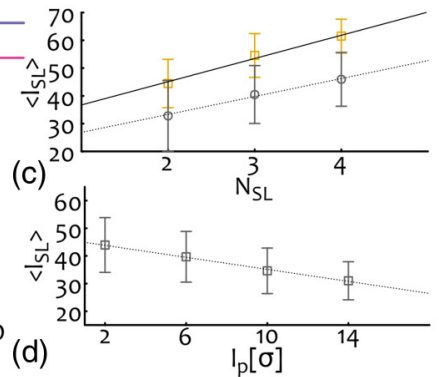

$I_{p}[\stackrel{10}{\sigma}]$

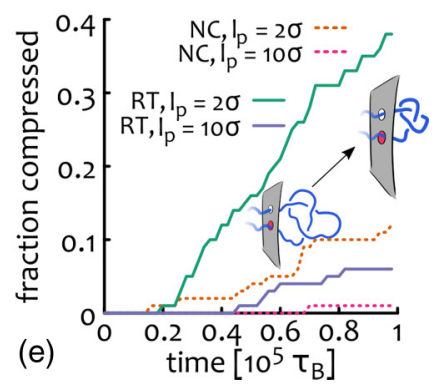

FIG. 4. Entropic Competition between SLs and a Trefoil Knot. (a) Plot of the distribution of lengths of the side with the SLs, for different SL number and topology. The plot shows that the RT configurations (solid lines) favor longer SL sides and hence tighter knots than NC configurations (dashed lines). (b) Distribution of lengths of the side with the SLs for different substrate persistence lengths: the curves refer to the case of a RT configuration with two SLs. The plot shows that stiffer substrates favor longer knotted sides. (c) Mean length $\left(\left\langle l_{\mathrm{SL}}\right\rangle\right)$ and standard error of the SL side length as a function of SL number and topology. The solid and dashed lines are best linear fits with slopes 8.4(8) for RT and 6.5(6) for NC. (d) Mean length $\left(\left\langle l_{\mathrm{SL}}\right\rangle\right)$ and standard error of the SL side length as a function of substrate persistence length $l_{p}$. The dashed line is the best linear fit with slope $-1.09(5) l_{p}$. (e) Fraction of knots that have been compressed-i.e., confined within $20 \%$ of the overall ring contour length-during the course of a simulation starting from a situation in which $80 \%$ of the contour length is on the knotted side. Higher flexibility and RT configurations both favor knot localization. NC denotes necklace, RT denotes round-table, and SLs denotes slip-links.

We employ Eq. (7) to derive semianalytically the distribution of loop lengths for systems with up to three SLs on each side. We then verify these predictions with molecular dynamics (MD) simulations and report the results in Fig. 3. Figure 3(a) shows that the distribution undergoes a unimodalbimodal transition for increasing ring sizes (we consider 35and 100-bead-long polymers). This transition can be viewed as a pitchfork bifurcation (see Appendix B), and is analogous to the symmetry-breaking behavior found with phantom chains in $[38,39]$.

We now turn to the case of two and three SLs on each side [Figs. 3(b)-3(d)]. We find that the spontaneous symmetry breaking is much more noticeable in the $\mathrm{NC}$ topology than in the RT one [Figs. 3(b) and 3(c)]. Finally, the RT topology displays a stable symmetric state for all polymer lengths when $N_{\mathrm{SL}}=3$ SLs are loaded on each side [Fig. 3(d)] with a remarkable absence of symmetry breaking. The histograms computed numerically in Figs. 3(b)-3(d) nicely match the predicted distributions in most cases. The one exception is the 2 SL RT case, where a third peak seems to appear for large $N$ in the simulated distributions: it would be of interest to study longer polymers to understand this case in more depth.

In light of these results, we argue that the network topology arising from the loading of multiple SLs - either in series (RT) or in parallel (NC) — profoundly affects the spatial organization of the underlying DNA or synthetic polymer. Further, our semianalytical results are in line with previous works, which showed that nested SLs (NC topology) promote growth, or extrusion, of the outer loop via a ratcheting process in which the diffusive dynamics of the outer loop is rectified by the entropic pressure of the inner ones [23].

\section{SLS VERSUS KNOTS}

The results of the previous section suggest that entropic competition of SLs can regulate the distribution of loop lengths of an underlying polymer. We now ask whether this entropic competition may also provide a mechanism for the localization and topological simplification of entanglements such as knots that may occur on biological or synthetic polymers. To address this question, we now consider the case in which the competing topologies are a SL network and a trefoil $\left(3_{1}\right)$ knot (as in Fig. 1), and we aim to identify the optimal conditions to localize and simplify the knotted loop. It should be noted that our calculations are performed in thermal equilibrium, so we seek entropic and topological mechanisms that bias the free energy towards a lower knotting probability. This is different from previous works, which studied the effects of energy-consuming mechanisms driving unknotting [32].

Since the semianalytical approach we used in the previous sections cannot be easily extended to this setup [44], we directly perform MD simulations of a 100-bead-long ring and address how different factors such as SL number, topology, and polymer persistence length affect the entropic competition and, in turn, regulate the probability and efficiency of knot localization.

First, we find a large difference between RT versus NC configurations [Fig. 4(a)]: the larger sliding entropy and low looping cost of the RT setup provide an entropic pressure that outcompetes the ratcheting effect of the $\mathrm{NC}$ topology. This result is unexpected, since it is known that $\mathrm{NC}$ configurations promote asymmetric polymer conformations and the formation of large loops with respect to RT networks [see Figs. 3(b) and 3(c) and Ref. [23]]. Nevertheless, the RT arrangement appears to be more suited to overcoming the knot entropy. Quantitatively, the entropic pressure of the RT configurations is notable: already with three SLs the knotted side is localized to a shorter contour length than the SL side [Fig. 4(a)].

Additionally, our simulations reveal that the bending rigidity of the polymer substrate also plays an important role [Fig. 4(b)]. Indeed, there are two potential and contrasting enthalpic effects that the bending rigidity may have on the entropic competition: on the one hand, the larger the stiffness, the longer the contour length required to form a knot without tight bends (this effect is particularly relevant for short loops); on the other hand, bending rigidity enhances the formation of large loops via diffusive loop extrusion [42]. Our simulations show that-for two SLs in a RT arrangement and a 


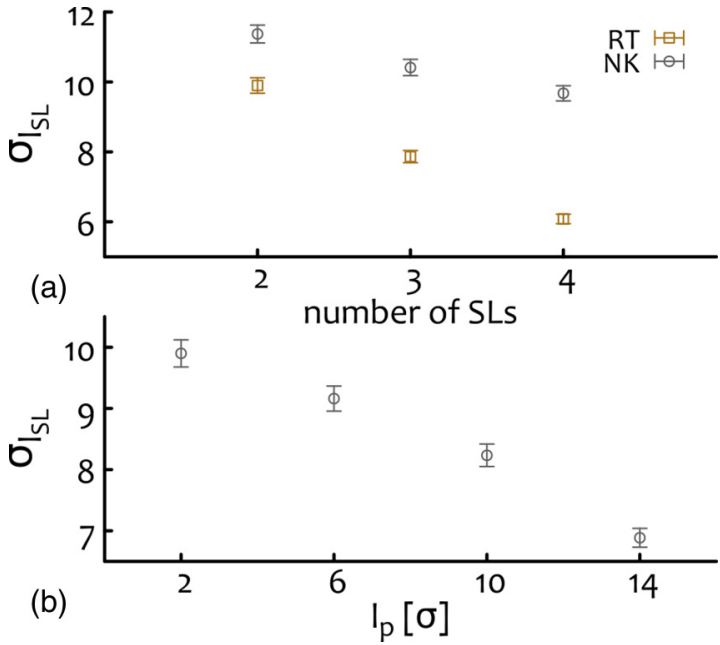

FIG. 5. Standard deviation of the SL side length $\left(\sigma_{l_{\mathrm{SL}}}\right)$ in the competition between SLs and a trefoil knot as a function of (a) SL number and topology, and (b) the persistence length $l_{p}$ of the substrate.

100-bead polymer ring-the former effect dominates: the larger the persistence length, the larger the knotted side, rendering the localization of entanglements less efficient [Fig. 4(b)]. This result shows that the entropic pressure provided by the SLs can only provide localization if the polymer substrate is sufficiently flexible.

More quantitatively, in Fig. 4(c) we show that the mean length of the SL-side, $\left\langle l_{\mathrm{SL}}\right\rangle$, grows linearly as a function of the number of SLs (in the range of $N_{\mathrm{SL}}$ explored here) and that the growth rate is faster for RT configurations. This linear behavior can be predicted asymptotically, up to corrections, by simplifying the scaling contribution of the SLs to account for the sliding entropy exclusively, and by assuming the nonuniversal connectivity constant to be topology-dependent (see Appendix B). At the same time, $\left\langle l_{\mathrm{SL}}\right\rangle$ appears to also decrease linearly with polymer stiffness as $\sim l_{0}-l_{p}$, with $l_{0}$ a constant [Fig. 4(d)]; this is expected, since the minimum number of monomers required to tie a knot with a given topology increases linearly with $l_{p}$ (e.g., in a freely jointed chain). In a practical genomic or synthetic context, the results reported in Figs. 4(a)-4(d) overall suggest that local SL density and substrate flexibility may provide handles to tune the typical size of knots. In vivo both of these parameters depend on local DNA sequence and transcriptional activity, and we thus speculate that knot and entanglement localization may be achieved to a different extent in different genomic regions; in particular, transcriptionally active genes (which are also thought to be more flexible) may harbor smaller knots and localized entanglements. At the same time, different synthetic polymers having different stiffness and different arrangements of cyclodextrins may be designed so that the resulting crosslinked gels have tunable properties [36].

We conclude this section by showing, in Fig. 5, that also the width of the distribution of lengths of the SL side is affected by the SL number, topology, and by the persistence length of the substrate. One can appreciate that as the number of SLs increases, so do the minimum number of monomers of the SL side and the entropic pressure exerted on the knotted side; consequently, the distribution of lengths of the SL side narrows. On the other hand, the minimum number of monomers involved in the knotted portion of a polymer ring increases with its persistence length, and thus the width of the distribution of lengths of the SL side decreases for stiffer substrates.

\section{KINETICS OF TOPOLOGICAL SIMPLIFICATION}

Another important aspect of the problem is how fast knots can be localized, and eventually removed. To measure the kinetics of knot localization, we perform MD simulations in which the system is initialized far from equilibrium with a large knot-occupying about $80 \%$ of the total contour length - and we measure how long it takes for the system to revert the situation and compress the knotted size to $20 \%$ of the total contour length. Figure 4(e) shows that RT configurations and flexible substrates are faster at localizing the knot, on top of being more efficient in steady state, as discussed before.

Finally, to address the question of whether diffusing SLs can drive the topological simplification of knots and entanglements, we now include topoisomerase-mediated strandcrossing reactions in our model as follows. We study a system in which a short segment of the knotted side is allowed to undergo strand-crossings. We then initialize the system with a given knot (either a 7-crossings torus knot 71 , a 10-crossings torus knot $10_{124}$, or a twist knot $7_{2}$ ) taking up $75 \%$ of the total contour length on the right-hand side, and we load SLs in RT arrangements on the other side. We chose to set only a short segment close to the wall as crossable to model the presence of a topoisomerase closely upstream of a SL-like SMC protein, as suggested by recent experimental evidence $[45,46]$ (note that in this context, we view the wall in Fig. 1 as a SL itself).

With this setup, we observe that the more SLs are loaded onto the substrate, the faster the rate of the topological simplification [see Fig. 6(a)]. The quantitative speedup is striking, as, regardless of the topology of the knot, the rate grows exponentially with $N_{\mathrm{SL}}$ [Fig. 6(b)]. An explanation of this is that the mean length of the SL side scales linearly with the number of SLs [Fig. 4(c)], so that the mean knotted length is $\left\langle l_{\mathcal{K}}\right\rangle=$ $L-\left\langle l_{\mathrm{SL}}\right\rangle \simeq L-k N_{\mathrm{SL}}$. Since the probability of observing a trivial knot on a polymer $x$ segments long scales as $P_{0}(L) \sim$ $e^{-x / x_{0}}[47,48]$, with $x_{0}$ a model-specific parameter, we expect $P_{0}\left(\left\langle l_{\mathcal{K}}\right\rangle\right) \sim e^{-\left\langle l_{\mathcal{K}}\right\rangle / L_{0}} \sim e^{N_{\mathrm{SL}} / L_{0}}$. In other words, as the knot size becomes smaller, its entropic cost increases sharply and it becomes exponentially harder to prevent its simplification to the unknot. (Note that this argument implicitly assumes that simplification is slow with respect to localization.)

\section{CONCLUSIONS}

In summary, in the context of a search for possible mechanisms of topological regulation and simplification of entanglements in DNA and other polymeric systems, here we have investigated the interplay between the entropy of SL networks and of knots.

In the case in which no knots are tied on the ring (the SLonly case, Fig. 3), we provide semianalytical predictions for the distribution of polymer lengths that compare well to MD 


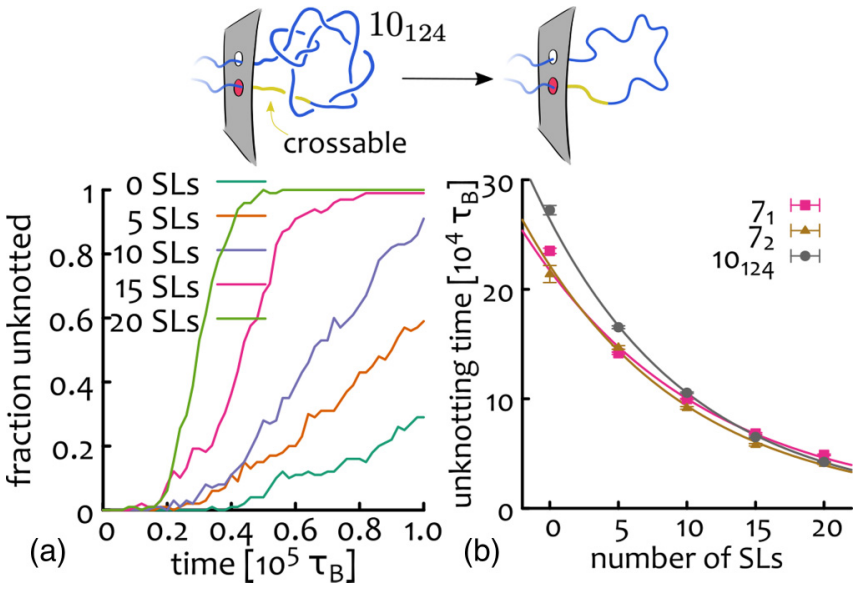

FIG. 6. Kinetics of topological simplification. (a) Fraction of $10_{124}$ knots that have simplified to unknots during the course of simulations of a polymer with $N=400$ beads, starting from a situation in which $80 \%$ of the contour length is on the knotted side. In this simulation, the 10 closest beads to the bead fixed at the wall on the knotted side are used to allow strand-crossings to model topoisomerase action to a slip link (SL). Loading more SLs increases the pressure on the knot and hence the rate of unknotting. (b) For all the knot topologies considered $\left(7_{1}, 7_{2}, 10_{124}\right)$, the simplification time (the time it takes to simplify a knot to the unknot with unitary probability) shows an exponential decrease as a function of the number of SLs. For the $10_{124}$, rates are computed by using the curves shown in (a).

simulations. We then consider a setup in which an SL network competes entropically with a trefoil knot, and we find that the network can localize the knot efficiently by just relying on its higher entropic pressure and in the absence of any motor activity associated with the SLs (such an activity is known to be absent in yeast cohesin [26] and cyclodextrins [36]). We dissect the effects of the number of SLs, the topology of the SL network, and the polymer flexibility on the knot localization discovering that round-table (or "in series") SL arrangements are best suited to confining a knot. Additionally, when the substrate is stiff, the enthalpic contribution from the bending energy dominates and the knot swells, hampering localization. Finally, we have also included topoisomerase in the model, which is relevant to study topological simplification of the genomes of living cells [32] or self-healing gels of polyrotaxanes with reversible bonds [49]. Strikingly, we find that entropic pressure alone is sufficient to simplify very complicated knots reliably and extremely fast, with the simplification rate increasing exponentially with the number of SLs.

We hope our findings may help unveil the mechanisms of topological simplifications of DNA by means of SL-like proteins such as SMC. Our results may also inform strategies for regulating the topology of synthetic polymer networks, for instance by using SLs to control the size of knots inside them, which can in turn affect their material properties.

\section{ACKNOWLEDGMENTS}

DMi is a Royal Society University Research Fellow; this work was supported by the Leverhulme Trust (ECF-2019088) and ERC (TAP, 947918).

\section{APPENDIX A: EQUATIONS FOR SLS VERSUS SLS USING DUPLANTIER SCALING FOR POLYMER NETWORKS}

The number of configurations of an asymptotically long monodisperse-i.e., where branches are equal in lengthpolymer network of total length $L$ and topology $\mathcal{G}$ in the dilute regime scales as [43]

$$
\mathcal{Z}_{\mathcal{G}}(L) \sim \mu^{L} L^{\gamma_{\mathcal{G}}-1}
$$

where

$$
\gamma_{\mathcal{G}}-1=-v d \mathcal{L}+\sum_{M \geqslant 1} n_{M} \sigma_{M}
$$

is a topology-dependent exponent: $d$ is the dimension, $\mathcal{L}$ is the number of loops, $n_{M}$ is the number of vertices of multiplicity (i.e., the number of branches connected to the vertex), and $M$ and $\sigma_{M}$ are the star polymer exponents.

If the network is grafted to a surface, Eq. (A1) changes to [43]

$$
\mathcal{Z}_{\mathcal{G}}^{S}(L) \sim \mu^{L} L^{\gamma_{\mathcal{G}}^{S}-1}
$$

where

$$
\gamma_{\mathcal{G}}^{S}-1=-v\left(d \mathcal{L}+\mathcal{V}_{\mathcal{S}}-1+\mathcal{L}_{S}\right)+\sum_{M \geqslant 1}\left(n_{M} \sigma_{M}+n_{M}^{S} \sigma_{M}^{S}\right)
$$

$n_{M}^{S}$ is the number of surface vertices (those grafted to the surface) of multiplicity $M, \mathcal{V}_{\mathcal{S}}=\sum_{M \geqslant 1} n_{M}^{S}$ is the number of surface vertices, $\mathcal{L}_{S}=\sum_{M \geqslant 1} M n_{M}^{S}$ is the number of branches connected to the surface, and $\sigma_{M}^{S}$ are the surface star polymer exponents.

\section{Surface loop and surface round-table exponents}

Let us call surface loop $O$ a simple loop with a single vertex grafted to a surface. A surface loop and the "surface" round table (RT) and necklace (with two slip-links only, NC) topologies we considered in our simulations (see Fig. 3) have only one surface vertex of multiplicity $2: \mathcal{V}_{S}=1$ and $\mathcal{L}_{S}=2$. According to Duplantier [43],

$$
\sigma_{2}^{S}=2 v-1,
$$

hence

$$
\gamma_{O}^{S}-1=-d v-1
$$

and

$$
\gamma_{\mathrm{RT}, N_{\mathrm{SL}}}^{S}-1=\gamma_{\mathrm{RT}, N_{\mathrm{SL}}}-2=-d v\left(N_{\mathrm{SL}}+1\right)+\sigma_{4} N_{\mathrm{SL}}-1,
$$

where $N_{\mathrm{SL}}$ is the number of slip-links,

$$
\gamma_{\mathrm{NC}, 2}^{S}=\gamma_{\mathrm{RT}, 2}^{S}
$$

\section{Surface round-table and necklace scaling with the lengths of the loops}

Analogously to what was predicted for the RT networks without a surface [16], we assume that the partition function 


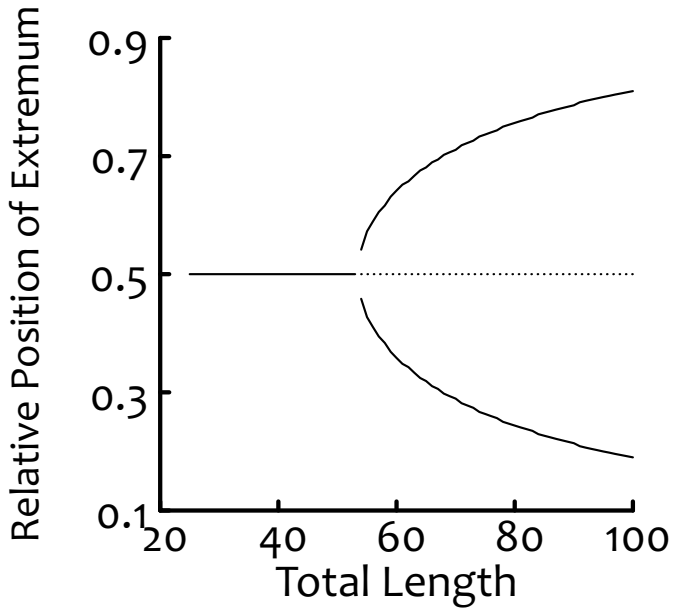

FIG. 7. Transition from unimodal to bimodal shape in the distribution of lengths of two slip-links competing with each other as predicted by Eq. (B1). The black solid lines are the positions of the stable points in the length distribution of one side of a polymer loop loaded with two competing slip-links. Dashed lines represent the unstable points.

of the surface RT networks with $N_{\mathrm{SL}}$ slip-links scales as

$$
\begin{aligned}
& \mathcal{Z}_{\mathrm{RT}, N_{\mathrm{SL}}}(L) \sim \\
& \sim \mu^{L} A\left(s_{1}, \ldots, s_{N_{\mathrm{SL}}}, L\right)\left(L-\sum_{i=1}^{N_{\mathrm{SL}}} s_{i}\right)^{\gamma_{\mathrm{RT}, N_{\mathrm{SL}}}^{S}-1} \\
& \mathcal{X}\left(\frac{s_{1}}{L-\sum_{i=1}^{N_{\mathrm{SL}}} s_{i}}, \ldots, \frac{s_{N_{\mathrm{SL}}}}{L-\sum_{i=1}^{N_{\mathrm{SL}}} s_{i}}\right)
\end{aligned}
$$

where with $s_{i}$ we indicate the lengths of the loops subtended by the slip-links (slip-linked loops), and $A\left(s_{1}, \ldots, s_{N_{\mathrm{SL}}}, L\right)$ accounts for the sliding entropy of the slip-links.

\section{Round table}

We now suppose the peripheral slip-linked loops are much smaller than the internal loop, as we see in our simulations. They contribute equally to Eq. (A9):

$$
\begin{aligned}
\mathcal{Z}_{\mathrm{RT}, N_{\mathrm{SL}}}(L) & \sim \\
\sim & \mu^{L} A\left(s_{1}, \ldots, s_{N_{\mathrm{SL}}}, L\right)\left(L-\sum_{i=1}^{N_{\mathrm{SL}}} s_{i}\right)^{\gamma_{\mathrm{RT}, N_{\mathrm{SL}}}^{S}-1} \\
& \times \prod_{i=1}^{N_{\mathrm{SL}}}\left(\frac{s_{i}}{L-\sum_{j=1}^{N_{\mathrm{SL}}} s_{j}}\right)^{\alpha}
\end{aligned}
$$

Furthermore, on the basis of this assumption, we require the scaling contribution of the largest loop to be that of a surface loop (without considering the sliding entropy; see [16] for the case without surface). This sets $\alpha$ to

$$
\alpha=\frac{\gamma_{\mathrm{RT}, N_{\mathrm{SL}}}^{S}-1}{N_{\mathrm{SL}}}-\frac{-d v-1}{N_{\mathrm{SL}}}=-d v+\sigma_{4},
$$

which is the same loop closure factor for the RT topologies without a surface. Finally,

$$
\begin{aligned}
& \mathcal{Z}_{\mathrm{RT}, N_{\mathrm{SL}}}(L) \sim \\
& \mu^{L} A\left(s_{1}, \ldots, s_{N_{\mathrm{SL}}}, L\right)\left(L-\sum_{i=1}^{N_{\mathrm{SL}}} s_{i}\right)^{-d v-1} \prod_{i=1}^{N_{\mathrm{SL}}} s_{i}^{-d v+\sigma_{4}} .
\end{aligned}
$$

\section{Surface necklace with two SLs}

In the case of the necklace topology with two slip-links, we write

$$
\begin{aligned}
& \mathcal{Z}_{\mathrm{NC}, 2}(L) \sim \\
& \sim \mu^{L} B\left(s_{1}, s_{2}, L\right)\left(L-s_{1}-s_{2}\right)^{\gamma_{\mathrm{NC}, 2}^{S}-1} \\
& \quad \times\left(\frac{s_{1}}{L-s_{1}-s_{2}}\right)^{\alpha_{1}}\left(\frac{s_{2}}{L-s_{1}-s_{2}}\right)^{\alpha_{2}},
\end{aligned}
$$

where we labeled as 1 the slip-linked loop grafted to the wall, and where $B\left(s_{1}, s_{2}, L\right)$ accounts for the sliding entropy. This time, we cannot state $\alpha_{1}=\alpha_{2}$ a priori. However, if we require the scaling contribution of the internal loop (which we assume to be the largest) to be that of a surface loop, and the contribution of the sliding slip-linked loop to be the usual closure factor [Eq. (A11)], the final result is

$\mathcal{Z}_{\mathrm{NC}, 2}(L) \sim \mu^{L} B\left(s_{1}, s_{2}, L\right)\left(L-s_{1}-s_{2}\right)^{-d \nu-1} s_{1}^{-d \nu+\sigma_{4}} s_{2}^{-d \nu+\sigma_{4}}$.

Notice that the consistency of Eqs. (A12) and (A14) with the hypothesis of the peripheral loops being small depends on the form of the sliding entropy terms $A$ and $B$. For the surface necklace, this might be a point of discussion; nonetheless, Eq. (A14) represents our simulated data reasonably well [see Fig. 2(b) in the main text].

\section{APPENDIX B: SCALING BEHAVIORS FOR COMPETING SLS}

In this Appendix, we list the formulas characterizing the scaling of the number of configurations of our system for different topologies.

The number of configurations of two slip-links competing with each other [Fig. 3(a)] scales as

$$
\mu^{L} \int_{s_{1}^{m}}^{l-s_{2}^{m}-s_{3}^{m}} d s_{1}\left(l-s_{2}^{m}-s_{3}^{m}\right) \frac{s_{1}^{-3 v+\sigma_{4}}}{\left(l-s_{1}\right)^{3 v+1}} \int_{s_{1}^{m}}^{L-l-s_{2}^{m}-s_{3}^{m}} d s_{1}\left(L-l-s_{2}^{m}-s_{3}^{m}\right) \frac{s_{1}^{-3 v+\sigma_{4}}}{\left(L-l-s_{1}\right)^{3 v+1}},
$$

where the coefficients $s_{i}^{m}$ account for the physical size of the slip-links: they are the minimum size of the segments of the slip-linked polymer; $L$ and $l$ are, respectively, the total length and the length of one side of the polymer. Note that the integrals account for the sliding entropy of the slip-links.
Figure 7 predicts the unimodal to bimodal transition seen in the histograms of Fig. 3(a); it is obtained numerically from Eq. (B1).

The number of configurations of two consecutive sliplinks competing with two consecutive slip-links [Fig. 3(c)] 
scales as

$$
\begin{aligned}
& \mu^{L} \int_{s_{1}^{m}}^{l-s_{2}^{m}-s_{3}^{m}-s_{4}^{m}-s_{5}^{m}} d s_{1} \\
& \quad \times \int_{s_{2}^{m}}^{l-s_{1}-s_{3}^{m}-s_{4}^{m}-s_{5}^{m}} d s_{2}\left(l-s_{1}-s_{2}-s_{3}^{m}-s_{4}^{m}-s_{5}^{m}\right)^{2} \frac{s_{1}^{-3 v+\sigma_{4}} s_{2}^{-3 v+\sigma_{4}}}{\left(l-s_{1}-s_{2}\right)^{3 v+1}} \\
& \quad \times \int_{s_{1}^{m}}^{L-l-s_{2}^{m}-s_{3}^{m}-s_{4}^{m}-s_{5}^{m}} d s_{1} \int_{s_{2}^{m}}^{L-l-s_{1}-s_{3}^{m}-s_{4}^{m}-s_{5}^{m}} d s_{2}\left(L-l-s_{1}-s_{2}-s_{3}^{m}-s_{4}^{m}-s_{5}^{m}\right)^{2} \frac{s_{1}^{-3 v+\sigma_{4}} s_{2}^{-3 v+\sigma_{4}}}{\left(L-l-s_{1}-s_{2}\right)^{3 v+1}} .
\end{aligned}
$$

\section{Average length of the SL side}

Let us call $\mathcal{Z}_{\mathrm{RT}, \tau}\left(l, L, N_{\mathrm{SL}}\right)$ the number of configurations of $N_{\mathrm{SL}}$ SLs in the RT configuration competing with a knot of topology $\tau$, with given $L$ and $l$, respectively, the total length of the system and the length of the SL side. Let us simplify the scaling of $\mathcal{Z}_{\mathrm{RT}, N_{\mathrm{SL}}, \tau}(l, L)$ as

$$
\mathcal{Z}_{\mathrm{RT}, \tau}\left(l, L, N_{\mathrm{SL}}\right) \simeq A \mu_{0}^{l} l^{N_{\mathrm{SL}}} l^{\alpha} \mu_{\tau}^{L-l} f(L-l, \tau),
$$

where $A$ is a constant amplitude, $\mu_{0}$ and $\mu_{\tau}$ are the connective constants of grafted knotted loops with topologies $\varnothing$ (unknotted) and $\tau, \alpha$ is the critical exponent $(\gamma-1)$ of the unknotted surface loops, and $f(L-l, \tau)$ is a function of the topology and length of the knotted side. $l^{N_{\mathrm{SL}}}$ accounts for the SLs, modeled as roots sliding along an unknotted loop. If we define

$$
F_{\mathrm{RT}, \tau}\left(N_{\mathrm{SL}}, L\right):=\int_{0}^{L} \mathcal{Z}_{\mathrm{RT}, \tau}\left(l, L, N_{\mathrm{SL}}\right) d l,
$$

then, if $\mu_{0} \neq \mu_{\tau}$, by integrating by part and imposing the boundary conditions,

$$
\begin{aligned}
& F_{\mathrm{RT}, \tau}\left(N_{\mathrm{SL}}+1, L\right) \simeq \frac{1}{a}\left(N_{\mathrm{SL}}+\alpha+1\right) F_{\mathrm{RT}, \tau}\left(N_{\mathrm{SL}}, L\right) \\
& +\frac{A \mu_{\tau}^{L}}{a} G_{\mathrm{RT}, \tau}\left(L, N_{\mathrm{SL}}\right),
\end{aligned}
$$

where

$$
G_{\mathrm{RT}, \tau}\left(L, N_{\mathrm{SL}}\right)=\int_{0}^{L} e^{-a l} l^{N_{\mathrm{SL}}+\alpha+1} \frac{\partial}{\partial l} f(L-l, \tau) d l
$$

and $a=\ln \frac{\mu_{\tau}}{\mu_{0}}$. Finally,

$$
\begin{aligned}
& \langle l\rangle_{\mathrm{RT}, \tau}\left(N_{\mathrm{SL}}, L\right)=\frac{F_{\mathrm{RT}, \tau}\left(N_{\mathrm{SL}}+1, L\right)}{F_{\mathrm{RT}, \tau}\left(N_{\mathrm{SL}}, L\right)} \\
& =\frac{1}{a}\left(N_{\mathrm{SL}}+\alpha+1\right)+\frac{A \mu_{\tau}^{L}}{a} \frac{G_{\mathrm{RT}, \tau}\left(L, N_{\mathrm{SL}}\right)}{F_{\mathrm{RT}, \tau}\left(N_{\mathrm{SL}}, L\right)} .
\end{aligned}
$$

Note that in the integration we have neglected the physical size of the SLs and the minimum number of monomers required to tie a knot of topology $\tau$.

For loops and links (not grafted) on the cubic lattice, it has been proven that $\mu_{0} \leqslant \mu_{\tau}$ [50-52]. It should be noted, however, that there is numerical evidence that $\mu_{0}$ and $\mu_{\tau}$ for these systems coincide to the second decimal place [50-52]. The calculations of this section can be extended to the case of two SLs in the NC configuration by replacing the sliding entropy factor $l^{N_{\mathrm{SL}}}=l^{2}$ with $l^{N_{\mathrm{SL}}-1}=l$.
[1] J. C. Turner and P. De Griend, History and Science of Knots, Series on Knots and Everything (World Scientific, Singapore, 1996).

[2] E. J. C. van Rijssel, J. B. Trimbos, and M. H. Booster, Mechanical performance of square knots and sliding knots in surgery: A comparative study, Am. J. Obstet. Gynecol. 162, 93 (1990).

[3] J. Chen, C. A. Rauch, J. H. White, P. T. Englund, and N. R. Cozzarelli, The topology of the kinetoplast DNA network, Cell 80, 61 (1995).

[4] S. A. Wasserman, J. M. Dungan, and N. R. Cozzarelli, Discovery of a predicted DNA knot substantiates a model for site-specific recombination, Science 229, 171 (1985).

[5] V. P. Patil, J. D. Sandt, M. Kolle, and J. Dunkel, Topological mechanics of knots and tangles, Science 367, 71 (2020).

[6] L. Postow, N. J. Crisona, B. J. Peter, C. D. Hardy, and N. R. Cozzarelli, Topological challenges to DNA replication: conformations at the fork, Proc. Natl. Acad. Sci. (USA) 98, 8219 (2001).
[7] A. D. Bates and A. Maxwell, DNA Topology (Oxford University Press, Oxford, 2005).

[8] J. M. Sogo, A. Stasiak, M. L. Martínez-Robles, D. B. Krimer, P. Hernández, and J. B. Schvartzman, Formation of knots in partially replicated DNA molecules, J. Mol. Biol. 286, 637 (1999).

[9] J. C. Wang, DNA topoisomerases, Annu. Rev. Biochem. 54, 665 (1985).

[10] M. Doi and S. F. Edwards, The Theory of Polymer Dynamics (Oxford University Press, Oxford, 1988).

[11] R. C. Ball, M. Doi, S. F. Edwards, and M. Warner, Elasticity of entangled networks, Polymer (Guildf.) 22, 1010 (1981).

[12] S. F. Edwards and T. Vilgis, The effect of entanglements in rubber elasticity, Polymer 27, 483 (1986).

[13] P. G. Higgs and R. C. Ball, Trapped entanglements in rubbers a unification of models, Europhys. Lett. 8, 357 (1989).

[14] D. Michieletto, On the tree-like structure of rings in dense solutions, Soft Matter 12, 9485 (2016).

[15] A. Y. Grosberg, Do knots self-tighten for entropic reasons? Polym. Sci.-Ser. A 58, 864 (2016). 
[16] R. Metzler, A. Hanke, P. G. Dommersnes, Y. Kantor, and M. Kardar, Tightness of slip-linked polymer chains, Phys. Rev. E 65, 061103 (2002).

[17] E. Orlandini, A. L. Stella, and C. Vanderzande, The size of knots in polymers. Phys. Biol. 6, 025012 (2009).

[18] A. E. Likhtman, The tube axis and entanglements in polymer melts, Soft Matter 10, 1895 (2014).

[19] G. Fudenberg, M. Imakaev, C. Lu, A. Goloborodko, N. Abdennur, and L. A. Mirny, Formation of chromosomal domains by loop extrusion, Cell Rep. 15, 2038 (2016).

[20] X. Wang, H. B. Brandão, T. B. K. Le, M. T. Laub, and D. Z. Rudner, Travel from origin to terminus, Science 355, 524 (2017).

[21] M. Ganji, I. A. Shaltiel, S. Bisht, E. Kim, A. Kalichava, C. H. Haering, and C. Dekker, Real-time imaging of DNA loop extrusion by condensin, Science 360, 102 (2018).

[22] I. F. Davidson, B. Bauer, D. Goetz, W. Tang, G. Wutz, and J.-M. Peters, DNA loop extrusion by human cohesin, Science 366, 1338 (2019).

[23] C. A. Brackley, J. Johnson, D. Michieletto, A. N. Morozov, M. Nicodemi, P. R. Cook, and D. Marenduzzo, Nonequilibrium Chromosome Looping Via Molecular Slip Links, Phys. Rev. Lett. 119, 138101 (2017).

[24] I. F. Davidson, D. Goetz, M. P. Zaczek, M. I. Molodtsov, P. J. Huisin 't Veld, F. Weissmann, G. Litos, D. A. Cisneros, M. OcampoHafalla, R. Ladurner, F. Uhlmann, A. Vaziri, and J.-M. Peters, Rapid movement and transcriptional relocalization of human cohesin on DNA, EMBO J. 35, 2671 (2016).

[25] J. Stigler, G. Ö. Çamdere, D. E. Koshland, and E. C. Greene, Single-molecule imaging reveals a collapsed conformational state for DNA-bound cohesin, Cell Rep. 15, 988 (2016).

[26] J.-K. Ryu, C. Bouchoux, H. W. Liu, E. Kim, M. Minamino, R. de Groot, A. J. Katan, A. Bonato, D. Marenduzzo, D. Michieletto, F. Uhlmann, and C. Dekker, Bridging-induced phase separation induced by cohesin SMC protein complexes, Sci. Adv. 7, eabe5905 (2021).

[27] K. Nasmyth, Disseminating the genome: Joining, resolving, and separating sister chromatids during mitosis and meiosis, Annu. Rev. Genet. 35, 673 (2001).

[28] M. Hirano and T. Hirano, Hinge-mediated dimerization of SMC protein is essential for its dynamic interaction with DNA, EMBO J. 21, 5733 (2002).

[29] J. H. Gibcus, K. Samejima, A. Goloborodko, I. Samejima, N. Naumova, J. Nuebler, M. T. Kanemaki, L. Xie, J. R. Paulson, W. C. Earnshaw, L. A. Mirny, and J. Dekker, A pathway for mitotic chromosome formation, Science 359, eaao6135 (2018).

[30] A. Goloborodko, J. F. Marko, and L. A. Mirny, Chromosome compaction by active loop extrusion, Biophys. J. 110, 2162 (2016).

[31] E. Piskadlo and R. A. Oliveira, A topology-centric view on mitotic chromosome architecture, Int. J. Mol. Sci. 18, 2751 (2017).

[32] E. Orlandini, D. Marenduzzo, and D. Michieletto, Synergy of topoisomerase and structural-maintenance-of-chromosomes proteins creates a universal pathway to simplify genome topology, Proc. Natl. Acad. Sci. (USA) 116, 8149 (2019).

[33] Y. Inoue, M. Miyauchi, H. Nakajima, Y. Takashima, H. Yamaguchi, and A. Harada, Self-threading of a poly(ethylene glycol) chain in a cyclodextrin-ring: control of the exchange dynamics by chain length, J. Am. Chem. Soc. 128, 8994 (2006).
[34] R. S. Forgan, J. P. Sauvage, and J. F. Stoddart, Chemical topology: Complex molecular knots, links, and entanglements, Chem. Rev. 111, 5434 (2011).

[35] K. Ito, Novel cross-linking concept of polymer network: Synthesis, structure, and properties of slide-ring gels with freely movable junctions, Polym. J. 39, 489 (2007).

[36] A. Bin Imran, K. Esaki, H. Gotoh, T. Seki, K. Ito, Y. Sakai, and Y. Takeoka, Extremely stretchable thermosensitive hydrogels by introducing slide-ring polyrotaxane cross-linkers and ionic groups into the polymer network, Nat. Commun. 5, 5124 (2014).

[37] Y. Yasuda, M. Toda, K. Mayumi, H. Yokoyama, H. Morita, and K. Ito, Sliding dynamics of ring on polymer in rotaxane: A coarse-grained molecular dynamics simulation study, Macromolecules 52, 3787 (2019).

[38] J. Rieger, Unusual bifurcation in a constrained random walk system, J. Phys. A 21, L1085 (1988).

[39] J. U. Sommer, Degenerate ground states of simple sliplink systems, J. Chem. Phys. 97, 5777 (1992).

[40] M. B. Hastings, Z. A. Daya, E. Ben-Naim, and R. E. Ecke, Entropic tightening of vibrated chains, Phys. Rev. E 66, 025102(R) (2002).

[41] R. Zandi, Y. Kantor, and M. Kardar, Entropic competition between knots and slip-links, ARI, Bull. ITU 53, 6 (2003).

[42] A. Bonato, C. A. Brackley, J. Johnson, D. Michieletto, and D. Mareduzzo, Chromosome compaction and chromatin stiffness enhance diffusive loop extrusion by slip-link proteins, Soft Matter 16, 2406 (2020).

[43] B. Duplantier, Statistical mechanics of polymer networks of any topology, J. Stat. Phys. 54, 581 (1989).

[44] This is because the scaling of the number of configurations of a knotted loop is known only in the asymptotic limit of a tight knot on a large loop.

[45] L. Vian, A. Pekowska, S. S. P. Rao, D. Levens, E. Lieberman Aiden, R. Casellas, L. Vian, K.-R. Kieffer-Kwon, S. Jung, L. Baranello, S.-C. Huang, L. El Khattabi, M. Dose, N. Pruett, A. L. Sanborn, A. Canela, Y. Maman, A. Oksanen, W. Resch, $\mathrm{X}$. Li et al., The energetics and physiological impact of cohesin extrusion, Cell 173, 1165 (2018).

[46] L. Uusküla-Reimand, H. Hou, P. Samavarchi-Tehrani, M. V. Rudan, M. Liang, A. Medina-Rivera, H. Mohammed, D. Schmidt, P. Schwalie, E. J. Young, J. Reimand, S. Hadjur, A. C. Gingras, and M. D. Wilson, Topoisomerase II beta interacts with cohesin and CTCF at topological domain borders, Genome Biol. 17, 182 (2016)

[47] N. Pippenger, Knots in random walks, Discret. Appl. Math. 25, 273 (1989).

[48] C. Micheletti, D. Marenduzzo, E. Orlandini, and D. W. Summers, Knotting of random ring polymers in confined spaces, J. Chem. Phys. 124, 064903 (2006).

[49] M. Nakahata, S. Mori, Y. Takashima, H. Yamaguchi, and A. Harada, Self-healing materials formed by cross-linked polyrotaxanes with reversible bonds, Chem. 1, 766 (2016).

[50] E. Orlandini, M. C. Tesi, E. J. J. van Rensburg, and S. G. Whittington, Asymptotics of knotted lattice polygons, J. Phys. A 31, 5953 (1999).

[51] A. Bonato, E. Orlandini, and S. G. Whittington, Asymptotics of linked polygons, J. Phys. A 53, 385002 (2020).

[52] A. Bonato, E. Orlandini, and S. G. Whittington, Asymptotics of multicomponent linked polygons, J. Phys. A 54, 235002 (2021). 